\title{
Study Protocol of a Cross-Sectional Study to Assess the Oral Health of and Oral Health Care for Older People Who Regularly Visit the Dental Practice
}

\section{Bots-VantSpijker PC ${ }^{1,2^{*}}$, Schols JMGA ${ }^{1,3}$, van der Maarel-Wierink CD $^{1,4}$ and Bruers $\mathrm{JJM}^{2,5}$}

${ }^{1}$ Flemish-Netherlands Geriatric Oral Research Group (BENECOMO), Ghent, Belgium, Nijmegen, Netherlands

${ }^{2}$ Department of Social Dentistry and Behavioral Sciences, Academic Centre for Dentistry Amsterdam (ACTA), University of Amsterdam and Vrije Universiteit, Amsterdam, Netherlands

${ }^{3}$ Caphri/Department of Family Medicine and Health Service Research, Maastricht University, Maastricht, Netherlands

${ }^{4}$ Center for Special Care in Dentistry, Amsterdam, Netherlands

${ }^{5}$ Royal Dutch Dental Association (KNMT), Utrecht, Netherlands

*Corresponding author: Bots-VantSpijker PC, Department of Social Dentistry and Behavioural Sciences, Academic Centre for Dentistry Amsterdam (ACTA), University of Amsterdam and Vrije Universiteit, Amsterdam, Netherlands, Tel: +31205980246; E-mail: pcbots-vantspijker@acta.nl

Received: February 15, 2019; Accepted: February 23, 2019; Published: February 26, 2019

Copyright: (c) 2019 Bots-VantSpijker PC, et al. This is an open-access article distributed under the terms of the creative commons attribution license, which permits unrestricted use, distribution, and reproduction in any medium, provided the original author and source are credited.

\begin{abstract}
Introduction: Little is known about the oral health of community-dwelling older people, as well as about which oral health care services dentists provide to these older people. It is also unclear to what extent general health issues play a role regarding this group of persons when it comes to maintaining good oral health and regular dental visits. Furthermore, much is unclear about the type and amount of care dentists offer to these people, which treatment strategies they apply, and whether they experience any barriers. These uncertainties have led to a study into the need for care of community-dwelling older people in general dental practices in the Netherlands as well as into the actual care dentists provide to this group of citizens.
\end{abstract}

Materials and methods: This exploratory cross-sectional study will focus on the oral health of older people in the Netherlands who still visit the general dental practice on a regular basis. Additionally, an inventory will be made of their needs for oral health care and the care, which is subsequently provided by dentists based on these needs. This study is based on two groups of older people: persons aged 75 and older and persons between the age of 60 and 64. The study is carried out by conducting a sample survey among general dental practitioners in the Netherlands. They will be asked to prospectively select and describe one older patient and to ask this patient to complete a short questionnaire.

Results and Discussion: The insights that will be gained through this study can be used to optimize the organization and quality of oral health care that dental practices provide to community dwelling (frail) older people. It is also expected that this study will identify knowledge gaps with regard to oral health care to older people in general dental practice.

Keywords: Study protocol; Cross-sectional study; Communitydwelling older people; Oral health; Oral health care provision

Abbreviations: SES: Socioeconomic Status; ISCO: International Standard Classification of Occupations; WMO: Human Research Act; METc: Medical Ethics Assessment Committee; KNMT: Royal Dutch Dental Association; KBA Nijmegen: Knowledge Centre Nijmegen; ISO: International Organization for Standardization; FDI: World Dental Federation; GOHAI: Geriatric Oral Health Assessment Index

\section{Introduction}

As people age, they face increasing risk of chronic diseases which will, in turn result in an increasing frailty and a growing reliance on care [1-3]. Suffering from chronic diseases is also related to the development of oral health problems [4], as oral health can be adversely affected by, amongst other things the side effects of certain medications used for the treatment of chronic diseases [5]. Additionally, chronic diseases can hamper the maintenance of a good oral health and result in decrease of daily oral hygiene care and fewer visits to a dental practice.

Even though an increasing number of older people now retain part of their own teeth into old age, they are seeking professional oral health care less frequently than younger people [6]. A retrospective study in Sweden has shown that, over a period of 4 years, $10 \%$ of the older people aged 75 and older who still live independently did not visit a dental practice on a regular basis [7].

The reduced visits to a dental practice can be due to insufficient knowledge about the importance of a good oral health, the accessibility of the care, the costs, and a fear or reluctance to visit the dentist. Consequently, there can be other factors of a socio-demographical nature which can be an impediment for older people to visit a dentist, for instance, when they are living in a rural environment, when they are lower-educated, or when they are single, as well as psychosocial factors such as loneliness and being dependent on the help of others [8-12]. 
Up to now, studies into the oral health of older people mainly focused on the generally poor oral health of older people living in a nursing home $[13,14]$. A recent study into the state of the oral health at the time of admission to a nursing home, carried out by Hoeksema et al. [15], showed that $80 \%$ of the older people already suffered from moderately to considerably poor oral health at the time of admission. In many western countries, an increasing number of older people live independently, sometimes with the help of informal carers or professional home care. In 2016, six out of seven people of 80 years or older living in the Netherlands were living independently, and this number is expected to rise even further in the future [16]. With respect to their health issues, community-dwelling older people depend on primary health care services. This also includes oral health care, which is provided by dentists in collaboration with other oral health care professionals. It is known that dentists, when treating (frail) older people, tend to encounter certain barriers. They may be concerned about or not at all familiar with specific problems or diseases older people can suffer from and with the effect's polypharmacy can have on oral health and possible dental treatments. They may also find that providing oral health care to frail older people is a complex matter or they are insufficiently prepared and equipped for the provision of oral health care to the older people with disabilities. Lastly, the reimbursement for the provided care can be deemed too low $[17,18]$.

Little is known about the oral health of community-dwelling older people, as well as about which oral health care dentists provide to these older people [19-22]. In either way, taking everything into consideration, the oral health of community-dwelling (frail) older people can find itself under pressure. At the same time, there is little factual knowledge about the need for oral health care of the specific group of independently living older people who still visit the dentist. This also applies to the extent to which general health issues play a role in this group of people when it comes to maintaining a good oral health and regular dental visits. Furthermore, much is unclear about the kind and amount of care dentists offer to these people, which treatment strategies they apply, and whether they experience any barriers.

These uncertainties have led to this study into the need for care of community-dwelling older people in general dental practice in the Netherlands as well as into the care dentists provide to this target group. This article describes the setup of this cross-sectional study.

\section{Materials and Methods}

\section{Study design}

This exploratory cross-sectional study will focus on the oral health of older people in the Netherlands who still visit the dental practice on a rather regular basis. Additionally, an inventory will be made of their needs for oral health care and the care, which is subsequently provided by dentists based on these needs. This study is based on two groups of older people: persons aged 75 and older and persons between the age of 60 and 64. It is expected that people from the age of 75 may have to cope with their increasing vulnerability and frailty and, therefore, encounter more problems with regard to maintaining their oral health and that they will find it more difficult to visit a dentist. The study also includes older persons between the age of 60 and 64 in order to identify predictive symptoms of oral diseases and a person's behavior with regard to oral health at a later age. The study is carried out by using a sample of general dental practitioners in the Netherlands. They will be asked to prospectively select and describe one older patient and to ask this patient to complete a short questionnaire.

\section{Recruitment of dentists}

From the total number of over 8,650 dentists below the age of 65 who live and/or practice in the Netherlands, a group of 3,000 dentists will be selected through random sampling. In stages, these dentists will receive an informative letter about the study in which they are informed that they will be contacted by phone during evening hours within one week for further explanation about the study. During this conversation, it will be checked whether the dentist is an eligible candidate for the study, meaning that the dentist will be asked whether he/she would be willing to select and describe an older patient from his/her client base. All phone calls will be made by an independent research agency ('Third Party Research Institute'). At the time of the first round of phone calls, an informative article about the motivation, the objective, and the structure of the study is to be published in the Nederlands Tandartsenblad (Dutch Dentists Magazine). The recruitment procedure will be definitively established after the first forty conversations with randomly selected dentists have taken place.

The aim is to collect data from around 900 patient-dentist combinations. The focus of this study lies on people aged 75 and older. However, as already stated, the study also includes a group of persons between the age of 60 and 64 . This will be done with an approximate ratio of 2:1, which, in numbers, means a group of 600 and a group of 300 combinations. Figure 1 shows a flow chart of the recruitment process.

\section{Research instruments}

Two research instruments have been developed for this study: a registration form for the dentist and a patient questionnaire. Based on a literature study $[13,23,24]$ and clinical experience of a study group of 7 practicing dentists, the domains for the registration form for the dentists have been determined. In addition to medical anamnesis, dental anamnesis, and dental visits in the past, the form also includes the diagnosis(es) and executed treatment(s) during the most recent visit as well as the treatment strategy. Furthermore, the dentists will be asked to add some additional information about the dental practice such as the size and composition of the dental team. Table 1 shows an overview of the aspects that will be collected via the registration form. The registration form was evaluated in the same study group of practicing dentists. During the evaluation, it was assessed to what extent requested data are available in the dental records and how much time it takes to complete the registration form. Some of the questions were rephrased based on this evaluation. The registration form was then evaluated again by one of the dentists from this study group and subsequently approved by the researchers.

The patient questionnaire was also drafted based on a literature study and clinical experience of the researchers. The questionnaire includes questions on the persons' marital status and socioeconomic status (SES), and about smoking and the intake of alcohol. The other questions refer to medication, frailty, (oral) self-care, and dental visits, as well as to the state of the persons' oral health and possible needs or wishes regarding oral health. Table 2 shows an overview of the aspects that persons will be asked via the questionnaire. The establishment of the persons' socioeconomic status will be based on their highest level of education (low/intermediate/high) and/or on their most recent occupation according to the ISCO classification [25]. The ISCO 
Citation: Bots-VantSpijker PC, Schols JMGA, Maarel-Wierink CD, Bruers JJM (2019) Study Protocol of a Cross-Sectional Study to Assess the Oral Health of and Oral Health Care for Older People Who Regularly Visit the Dental Practice. J Gerontol Geriatr Res 8: 498. doi: $10.4172 / 2167-7182.1000498$

Page 3 of 5

classification distinguishes four skill levels. The questionnaire has been assessed on comprehensibility by 2 older persons and established by the researchers.

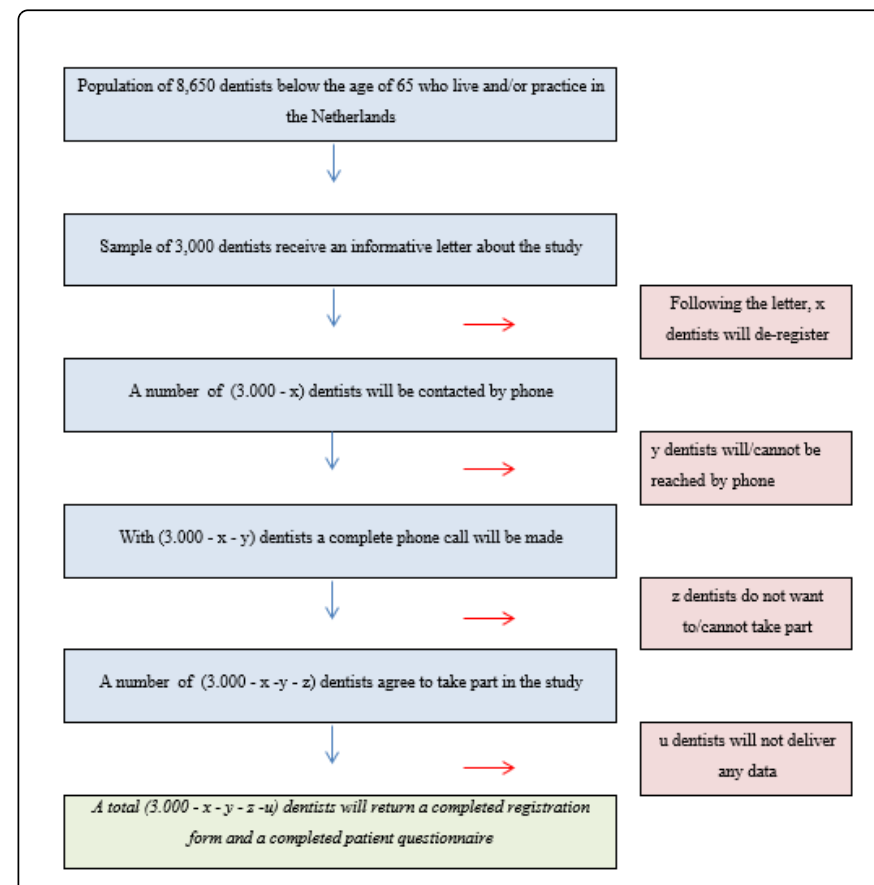

Figure 1: Flow chart of the recruitment process.

\begin{tabular}{|c|c|}
\hline S.no & Data recorded in the registration form \\
\hline 1 & $\begin{array}{l}\text { Medical anamnesis: Has a medical anamnesis been performed, and } \\
\text { if so, presents diseases and pathologies and other aspects ( } 3 \text { items). }\end{array}$ \\
\hline 2 & $\begin{array}{l}\text { Dental anamnesis: Status praesens, presence of complete or partial } \\
\text { maxillary and mandibular dentures and other aspects ( } 22 \text { items). }\end{array}$ \\
\hline 3 & $\begin{array}{l}\text { Dentist visits in the past, diagnoses and treatments: Number of dental } \\
\text { visits, reason and diagnosis most recent visit, type of oral health care } \\
\text { provided and other aspects ( } 7 \text { items). }\end{array}$ \\
\hline 4 & $\begin{array}{l}\text { Treatment policy: Presence of treatment strategy, whether or not } \\
\text { discussed with the patient and other aspects ( } 10 \text { items). }\end{array}$ \\
\hline 5 & $\begin{array}{l}\text { Characteristics dental practice: Characteristics of the dental team and } \\
\text { patient population, wheelchair accessibility and other aspects ( } 9 \\
\text { items). }\end{array}$ \\
\hline Note & nslated registration form is available from the first author \\
\hline
\end{tabular}

Table 1: An overview of aspects of oral health and oral health care of an older person, about which dentists were asked to record data in the registration form.

\section{Data collection}

Dentists who agree to take part in the study will receive a package with the required documents and information for the involvement of a patient who is either aged 75 or older or between the age of 60 and 64. The package includes an instruction leaflet for the dentist, a letter containing information for the patient, two informed consent forms, a registration form for the dentist, and the patient questionnaire. When necessary, the dentists may receive letters to remind him to collaborate with the study, which will be sent 1 and 2 months respectively after the packages have been sent. Should a third reminder be required, then the dentists who have not yet responded will be sent a letter in which they are informed that they will also be called to remind them of their participation. These dentists will receive a phone call at their practice with a last request to participate in this study. In order to get an impression as to why these dentists do not or no longer wish to respond, an inventory of the reasons for not delivering the requested information will be made as well. Then the data collection period will be closed.

\begin{tabular}{|c|c|}
\hline S.no & Data recorded in the questionnaire \\
\hline 1 & $\begin{array}{l}\text { General information: Age, marital status, education and other aspects } \\
\text { ( } 7 \text { items). }\end{array}$ \\
\hline 2 & $\begin{array}{l}\text { Health and frailty: Smoking and drinking behavior, medication use, } \\
\text { being able move, being able to take care of oneself and other aspects } \\
\text { (11 items). }\end{array}$ \\
\hline 3 & $\begin{array}{l}\text { Oral health selfcare: Tooth brushing, interdental cleaning, } \\
\text { independently or with help and other aspects ( } 4 \text { items). }\end{array}$ \\
\hline 4 & $\begin{array}{l}\text { Oral function: Chewing and swallowing, pain and other oral complaints } \\
\text { ( } 9 \text { items). }\end{array}$ \\
\hline 5 & $\begin{array}{l}\text { Dental visits: Frequency of visits, possible barriers related to and } \\
\text { accessibility of the dental practice, reason for dentist visit and other } \\
\text { aspects ( } 8 \text { items). }\end{array}$ \\
\hline & \\
\hline
\end{tabular}

Table 2: An overview of aspects of oral health and oral health care, about which an older person was questioned on in the questionnaire.

\section{Patient selection procedure}

With respect to the selection of an older person, the dentist will be asked to take the first prospective patient, according to his appointment schedule, who is either aged 75 or older or who is between the age of 60 and 64 respectively. The person can be included when he has been seen by the dentist for a check-up or treatment within the last 2 years. The person will be invited to participate in the study during the upcoming actual visit to the practice. When the person gives his consent, he will be handed the information letter with the request to read it carefully. When the person has read the information letter and agrees to cooperate, both the person and the dentist will sign the informed consent form in duplicate. With the consent form, the person declares that the participation is done voluntarily, that his answers to the questions can be used for this study, and that, for the purpose of this study, the person gives the dentist his consent to disclose to the researchers some anonymized data from his patient file (which will be coded). The consent form for the dentist states that the disclosure of data will take place in a careful and confidential manner.

A copy of the signed informed consent form will be handed to the person after which he is asked to complete the patient questionnaire. This can either be done before or after the person' consultation and, when necessary, with the help of the dental assistant. The questionnaire can also be taken home and, after completion, returned in the enclosed postage-paid envelope. During or after the consultation, the dentist can complete the dentist-registration form based on the patient file and the findings during the person' visit to the practice. The completed registration form, the informed consent form, and, when already 
completed, the patient questionnaire will then be returned to the researchers with the use of a postage-paid envelope.

\section{Data processing}

The data from the returned questionnaires and registration forms will be collected by the above-mentioned independent research agency and entered under code into data files with the use of entry screens that are specifically programmed for this purpose. When entering the data, the same code will be attributed to the data from the patient questionnaire and the corresponding registration form of the dentist. This will ensure that the data can be related to an individual patient, an individual dentist, and an individual patient-dentist combination. The researchers will then only obtain the files containing the coded research data in which the participating persons and dentists are in no way identifiable. The independent research agency shall retain the collected registration forms and patient questionnaires in order for them to be destroyed after the study has been completed.

The files, one with the collected data obtained via the completed dentist registration forms and one with the collected data obtained via the patient questionnaires, will first be checked on coding errors and on inconsistencies and missing data. When required, the (coded) returned forms and lists will be consulted for this purpose Subsequently, these separate files will be linked and processed into one research file based on the previously attributed codes.

\section{Ethics approval and consent to participate}

In the Netherlands, according to the Medical Scientific Research involving Human Subjects Act (WMO) for research in which research data are obtained by studying medical records, a non-WMO declaration is desirable. The METc of the Vrije Universiteit Amsterdam has provided such a non-WMO-declaration in July 2016. To guarantee the confidentiality for the processing of the data for this study and to safeguard the anonymity of the participants, an independent research agency ('Third Party Research Institute) was employed. Commissioned by the Royal Dutch Dental Association (KNMT), this agency, KBA in Nijmegen (KBA, 2018) takes care of the data collection and data processing for studies in dental practices. $\mathrm{KBA}$ is a member of a national Association for Policy Research [26], is ISO-certified (ISO-9001: 2008) and carries out the work activities in compliance with a legally mandatory data processing agreement.

\section{Statistical analysis}

Based on a description of the data on the oral health of older people, which will be partly collected via the persons themselves and partly via the participating dentists, analyses will be made of the need for oral health care of community-dwelling older people provided by general dental practices in the Netherlands as well as the type and amount of oral health care already provided by dentists to community-dwelling older people.

The starting point in the descriptions will be the definition of oral health which was recently formulated by the FDI World Dental Federation [27]. Furthermore, the comparison between the age groups of the people, the first one consisting of older persons between the age of 60 and 64 and the other one being the group of older persons aged 75 and older, will continuously be applied. Based on this comparison, conclusions will be formulated on the self-care of the older people, on their visits to the dental practice, their general health, their living conditions, the recently received care, and the other collected data. For the purpose of these descriptions, the usual statistical measures for distribution and dispersion will be applied. Where necessary, new variables may be construed via factor analysis, the establishment of sum scores, and/or via other ways later to be determined.

It will subsequently be assessed whether or not and if so, in what way the oral health of older people who still visit the dental practice is consistent with general or more specific patient characteristics and/or characteristics of the dentists by whom they are treated. Amongst other things, this may demonstrate whether older people with good oral health and older people with a poorer oral health can be identified as a group. Additionally, it may demonstrate whether possible predictive symptoms of oral diseases and behavior regarding oral health at a later age can be identified.

All data will be processed, linked, and analyzed with the use of SPSS statistic software (IMB Corp, 2016). During this process, appropriate procedures such as Chi-square test, t-test, ANOVA, regression, and others will be applied.

\section{Results}

Notwithstanding the unique character of this study, some limitations can be identified beforehand. One of the limitations is that it will be a challenge to achieve the intended and desired response of around 900 patient-dentist combinations. It is expected that not every dentist has the time and inclination to participate in this study and it is conceivable that a good number of dentists will be unable to recruit (at short notice) a suitable person who is willing to participate. It may also be possible that, despite a prior commitment to participate in the study, a dentist and/or patient will not deliver any data. Moreover, based on the cross-sectional design of the study, no causal relations can be examined.

\section{Discussion}

Whereas, until far into the $20^{\text {th }}$ century, the majority of older people in the Netherlands were entirely edentulous, older people nowadays still have (part of) their own teeth [28]. Many of them still have regular dentist appointments up to a very advanced age [29]. Nevertheless, little is known about the oral health of these community-dwelling and possibly frail older people and of the oral health care they are given. This article describes how one of the first descriptive studies into this subject has been set up. This also takes into account the general health of the older people, their living conditions and their requirements and needs regarding oral health. The fact that this issue is addressed from the perspective of both the dentist and the patient makes this study unique. This is because most studies in this area only describe the oral health of older people by means of self-reporting or by carrying out the GOHAI [8] or they are solely based on data disclosed by dentists $[9,13]$. The study protocol can be extended to a longitudinal version without much difficulty. In that case, it is necessary to measure the (changeable) items described here, repeatedly at the recruited dentistpatient couples. Obviously, these dentists and patients must be informed about this and must agree to this via informed consent.

\section{Conclusion}

The insights that will be gained through this study can be used to optimize the organization and quality of oral health care that dental practices provide to community-dwelling (frail) older people. This study is also expected to identify knowledge gaps with regard to 
Citation: Bots-VantSpijker PC, Schols JMGA, Maarel-Wierink CD, Bruers JJM (2019) Study Protocol of a Cross-Sectional Study to Assess the Oral Health of and Oral Health Care for Older People Who Regularly Visit the Dental Practice. J Gerontol Geriatr Res 8: 498. doi: $10.4172 / 2167-7182.1000498$

Page 5 of 5

providing oral health care to older people in the dental practice. The obtained information will then lead to insights that can be used for further studies.

\section{Conflicts of Interest}

There are no conflicts of interest for the present study.

\section{Availability of Data and Material}

All research instruments, letters and instructions are available on request via the corresponding author.

\section{Funding}

All costs related to the data collection in this study have been funded by the Royal Dutch Dental Association (KNMT).

\section{References}

1. Gobbens RJ, Van Assen MA, Luijkx KG, Schols JM (2012) The predictive validity of the tilburg frailty indicator: Disability, health care utilization, and quality of life in a population at risk. Gerontologist 52: 619-631.

2. Manton KG, Gu X, Lamb VL (2006) Change in chronic disability from 1982 to $2004 / 2005$ as measured by long-term changes in function and health in the US elderly population. Proc Natl Acad Sci 103: 18374-18379.

3. Ouchi Y, Rakugi H, Arai H, Akishita M, Ito H, et al. (2017) Redefining the elderly as aged 75 years and older: Proposal from the Joint Committee of Japan Gerontological Society and the Japan Geriatrics Society. Geriatr Gerontol Int 17: 1045-1047.

4. van Der Putten GJ, De Baat C, De Visschere L, Schols J (2014) Poor oral health: A potential new geriatric syndrome. Gerodontology 31: 17-24.

5. Ciancio SG (2004) Medications' impact on oral health. J Am Dent Assoc 135: 1440-1448.

6. Slack-Smith L, Lange A, Paley G, O'grady M, French D, et al. (2010) Oral health and access to dental care: A qualitative investigation among older people in the community. Gerodontology 27: 104-113.

7. Derblom C, Hagman-Gustafsson ML, Gabre P (2017) Dental attendance patterns among older people: A retrospective review of records in public and private dental care in Sweden. Int J Dent Hyg 15: 321-327.

8. Kiyak HA, Reichmuth M (2005) Barriers to and enablers of older adults' use of dental services. J Dent Educ 69: 975-986.

9. Brothwell DJ, Jay M, Schönwetter DJ (2008) Dental service utilization by independently dwelling older adults in Manitoba, Canada. J Can Dent Assoc 74: 161.

10. Borreani E, Wright D, Scambler S, Gallagher JE (2008) Minimising barriers to dental care in older people. BMC Oral Health 8: 7.

11. Manski R, Moeller J (2017) Barriers to oral health across selected European countries and the USA. Int Dent J 67: 133-138.

12. Niesten D, Witter DJ, Bronkhorst EM, Creugers NH (2017) Oral health care behavior and frailty-related factors in a care-dependent older population. J Dent 61: 39-47.

13. De Visschere L, Janssens B, De Reu G, Duyck J, Vanobbergen J (2016) An oral health survey of vulnerable older people in Belgium. Clin Oral Investig 20: 1903-1912.
14. Karki AJ, Monaghan N, Morgan M (2015) Oral health status of older people living in care homes in Wales. Br Dent J 219: 331-334.

15. Hoeksema AR, Peters LL, Raghoebar GM, Meijer HJ, Vissink A, et al. (2017) Oral health status and need for oral care of care-dependent indwelling elderly: From admission to death. Clin Oral Investig 21: 2189-2196.

16. CBS (2016) Huishoudensprognose 2015 - 2060: jongeren en ouderen langer thuis: 1-32. Available via: https://www.cbs.nl/-/media/_pdf/ 2016/24/2016-huishoudenprognose-2015-2060.pdf. (Article in Dutch). Accessed 26 February 2019.

17. Bots-VantSpijker PC, Bruers JJ, Bots CP, Vanobbergen JN, De Visschere LM, et al. (2016) Opinions of dentists on the barriers in providing oral health care to community-dwelling frail older people: A questionnaire survey. Gerodontology 33: 268-274.

18. Smith MB, Thomson WM (2017) 'Not on the radar': Dentists' perspectives on the oral health care of dependent older people. Gerodontology 34: 90-100.

19. Gluzman R, Meeker H, Agarwal P, Patel S, Gluck G, et al. (2013) Oral health status and needs of homebound elderly in an urban home-based primary care service. Spec Care Dent 33: 218-226.

20. Mei Na T, Nair R, Di Ying JN, Yee R (2014) Oral health status and complete denture status of independent-living Singaporean elderly residing in a community home. Singapore Dent J 35: 9-15.

21. Brennan DS, Keuskamp D, Balasubramanian M, Amarasena N (2018) General health and well-being among primary care patients aged 75+ years: Associations with living conditions, oral health and dependency. Australas J Ageing 37: E1-6.

22. Kohli R, Sehgal HS, Nelson S, Schwarz E (2017) Oral health needs, dental care utilization, and quality of life perceptions among Oregonian seniors. Spec Care Dent 37: 85-92.

23. Holmén A, Strömberg E, Hagman-Gustafsson ML, Wårdh I, Gabre P (2012) Oral status in home-dwelling elderly dependent on moderate or substantial supportive care for daily living: Prevalence of edentulous subjects, caries and periodontal disease. Gerodontology 29: e503-511.

24. Niesten D, Witter D, Bronkhorst E, Creugers N (2016) Oral health-related quality of life and associated factors in a care-dependent and a careindependent older population. J Dent 55: 33-39.

25. Classification of Occupations (2012) In: ILO ILO, International Standard Classification of Occupations ISCO-08, Geneva 1:2.

26. Bruers JJ, Boer JC, Van Dam BA (2014) The Data Stations project: a monitor of dental practice in The Netherlands. Ned Tijdschr Tandheelkd 121: 345-352. (Article in Dutch).

27. Glick M, Williams DM, Kleinman DV, Vujicic M, Watt RG, et al. (2016) A new definition for oral health developed by the FDI World Dental Federation opens the door to a universal definition of oral health. Int Dent J 66: 322-324.

28. CBS.https://www.cbs.nl/nl-nl/nieuws/2003/45/steeds-minder-mensenhebben-een-kunstgebit. Accessed 15 february 2019.

29. CBS.http://statline.cbs.nl/Statweb/publication/? $\mathrm{DM}=\mathrm{SLNL} \& \mathrm{PA}=83005 \mathrm{NED} \& \mathrm{D} 1=67-69 \& \mathrm{D} 2=0-13 \& \mathrm{D} 3=0 \& \mathrm{D} 4=1 \& \mathrm{HDR}$ $=\mathrm{G} 2, \mathrm{G} 3, \mathrm{~T} \& \mathrm{STB}=\mathrm{G} 1 \& \mathrm{VW}=\mathrm{T}$. Accessed 15 february 2019. 